\title{
Relacionalidad y construcción cultural en la educación temprana: diálogo entre pares menores de 18 meses $^{1}$
}

\author{
Eugenia Pizarro Troncoso ${ }^{2}$ \\ Sergio Toro Arévalo²
}

\section{Resumen}

El presente artículo da cuenta de una investigación realizada en nivel preescolar, específicamente en una sala cuna chilena. El objetivo de este estudio fue analizar el fenómeno de la relacionalidad y la construcción cultural que se despliega en la interacción con pares durante los primeros 18 meses de vida. Para esto se utilizó un enfoque cualitativo, recolectando datos mediante entrevistas, observación participante y análisis microgenético de diversas situaciones de interacción protagonizadas por bebés, para la posterior triangulación de fuentes. El estudio da cuenta de las dinámicas relacionales que se despliegan entre niñas y niños pequeños en la primera etapa de educación preescolar, desde una perspectiva cultural y dialógica de la construcción del sujeto. Los resultados se organizan en tres categorías de análisis denominadas miradas, resonancia gestual y mediación, las que dan cuenta de la riqueza y complejidad de los procesos de construcción cultural desde edades muy tempranas, donde destacan las diversas narrativas que se entretejen en la relación entre pares y su distinción con la relacionalidad adulta/bebé. Las conclusiones apuntan a la relevancia de las interacciones entre pares en la construcción de significados de cada bebé; al mismo tiempo, se discute la importancia de la mediación adulta en estas dinámicas relacionales. La contribución de esta investigación apunta a enriquecer el debate sobre la pertinencia y relevancia de la educación preescolar de niñas y niños menores de 3 años.

\section{Palabras clave}

Educación temprana - Primera infancia - Pares - Relaciones dialógicas.

\section{Relationality and cultural construction in early education: dialogue between peers under 18 months}

\footnotetext{
1- Investigación financiada por FONDECYT Regular n.1120761. 'Conocimiento y Comunidad Escolar: Procesos de Interpretación de las Dinámicas Relacionales Escolares en la Educación Básica como Configuración Cognitiva Situada', 2012-2014.

2- Universidad Austral de Chile, Valdivia, Chile. Contactos: eugeniapizarro@uach.cl; seatoro@gmail.com
}

DOl: http://dx.doi.org/10.1590/S1678-4634201706160928 


\section{Abstract}

This paper reports on research developed at the preschool level, specifically in a Chilean nursery school. The objective of this study was to analyze the phenomenon of relationality and cultural construction that unfolds in peer interaction during the first 18 months of life. With this purpose, a qualitative approach was used, collecting data through interviews, participant observation and microgenetic analysis of diverse interaction situations performed mainly by babies, followed by data source triangulation. This study reports on the relational dynamics that unfold between small girls and boys in the first stage of preschool education, from a cultural and dialogic perspective of subject construction. The results are organized in three analysis categories named gazes, gestural resonance and mediation. These categories evidence the richness and complexity of the cultural construction processes from very early ages, in which the diverse narratives intertwining in the peer relationship and their distinction with adult/baby relationality stand out. The final conclusions point to the relevance of peer interactions in the construction of meanings of each baby. At the same time, the importance of adult mediation in these relational dynamics is discussed. This researco enriches the debate about the appropriateness and relevance of preschool education for girls and boys under three years of age.

\section{Keywords}

Early education, early childhood - peers, dialogic relationships.

El ser humano se construye en relación con otro u otros sociales, con los objetos, con el espacio y con la mediación semiótica que cada uno de estos implica desde las primeras etapas de su vida. El presente documento comprende esta relacionalidad desde una perspectiva dialógica y cultural que se fundamenta, por una parte, en el concepto de relación dialógica, comprendido desde la propuesta bajtiana (BAJTÍN, 1999; SCORSOLINI-COMIN; SANTOS, 2010) y, por otra parte, en un enfoque cultural del desarrollo humano (VALSINER, 2000; 2001). Además, son consideradas propuestas que han aplicado estas teorías a los estudios de las primeras etapas del desarrollo humano (AMORIM; ROSSETTI-FERREIRA; VITORIA, 2000; AMORIM; ROSSETTI-FERREIRA, 2008a, 2008b; CORREIA, 2009).

Desde la perspectiva de las relaciones dialógicas, Bajtín (1999) propone comprender la comunicación como ejercicio donde tanto el hablante como el oyente son sujetos activos, insertos en un contexto cultural y social. Entendido desde este enfoque, las relaciones dialógicas se encuentran a la base del lenguaje, el sentido y el pensamiento (BAJTÍN, 1999). Esto supone, además, que la construcción del sujeto y de la cultura no se comprende sin la existencia de otro, sin el colectivo de los hablantes.

Esta relación dialógica, además, es entendida desde una dimensión histórica $\mathrm{y}$ situada, donde cada enunciado está cargado de ecos pertenecientes a otros enunciados, a las voces de otros, al mismo tiempo que es producido en un contexto específico, donde el enunciado se hace propio, cargado de una intención comunicativa determinada; en el 
mismo ejercicio, el enunciado es proyectado a futuro, es decir, busca la respuesta de otro u otros. En este marco, el enunciado es entendido como un signo ideológico que nunca es neutro, dado que está arraigado a sus contextos históricos, sociales y culturales de su producción (AMORIM; ROSSETTI-FERREIRA, 2008a; BAJTÍN, 1999; SCORSOLINI-COMIN; SANTOS, 2010).

De acuerdo con Valsiner (2000, 2001), el desarrollo humano es intrínsecamente cultural. En este sentido, cabe destacar que el autor comprende cultura como "mediación semiótica” (2000, p. 49), que funciona a nivel personal y colectivo. Esta mediación participa de la construcción del sujeto, quien va negociando significados con su entorno, lo que podría explicarse como una co-construcción individuo/cultura. Al mismo tiempo, la cultura se configura como herramienta de regulación social.

Desde esta perspectiva, la construcción del sujeto se desarrolla en relación con un medio físico y social organizado culturalmente, en un mundo que es heterogéneo en tiempo y espacio, y que es único para cada ser humano, presentando un escenario complejo. Este abordaje implica una lectura de las relaciones sociales que releva el contexto donde se despliegan, así como los significados que se construyen en estos contextos.

Amorim y Rossetti-Ferreira (2008a, 2008b), por otra parte, aplican la lectura dialógica y la perspectiva cultural del desarrollo, a los procesos relacionales que se despliegan en etapas preverbales. Esto implica una comprensión de la o el bebé como ser histórico, social, que participa activamente en su desarrollo, agenciando una rica variedad de contenidos socio-culturales y construyendo significados mediados culturalmente.

$\mathrm{Al}$ respecto, Correia (2009) propone que estos procesos de construcción de significados se encuentran presentes antes del uso de los signos específicos asociados a la lengua materna, de tal manera que no pueden ser considerados como una función o un elemento del lenguaje. Más aún, si bien la palabra está cargada de significado, no siempre logra comunicarlo todo, por el contrario, frecuentemente esconde o distorsiona este significado. De esta forma, el lenguaje se entiende como la expresión visible de los procesos de construcción de significados.

La construcción cultural, en este sentido, implica procesos de construcción de significados compartidos, a través de las interacciones con otro y otros sociales en un contexto determinado (CORREIA, 2009; VALSINER, 2000). Mediante este proceso el ser humano va tejiendo significados compatibles con su contexto social, necesarios para la comunicación, los que la o el bebé organiza en secuencias narrativas (CORREIA, 2009; BRUNER, 2006). Estas narrativas permiten dar sentido a la experiencia, interpretándola a partir del contexto donde son producidas. En el caso de los primeros años de vida, la construcción de narrativas se encuentra particularmente orientada la acción humana, donde resultan especialmente relevantes las consecuencias de dichas acciones. De esta forma, desde muy temprano el niño y la niña presentan una inclinación a atender a los eventos inusuales y dejar de marcar los habituales, lo que se refleja desde sus primeros intentos lingüísticos (BRUNER, 2006).

Por otra parte, Anjos y otros autores proponen la presencia de un "campo interactivo" (2004, p. 521), donde hay interés en el otro, y el comportamiento se regula a partir de las acciones del otro, ya sea que éste lo sepa o no. En este sentido, destacan la capacidad 
interactiva de las y los bebés, la que es apreciable desde el nacimiento. Al respecto, cabe destacar la relevancia de la corporiedad en la comprensión del desarrollo humano, que se entiende desde la noción de inseparabilidad biológico/cultural propuesta por Amorim y Rossetti-Ferreira (2008b). En este sentido, Anjos y otros autores (2004), relevan el lugar del cuerpo en la relación dialógica, señalando que a través de éste se vivencia el mundo y se construye la relación consigo mismo y con los otros sociales.

Bajo estos enfoques, la concepción tradicional de la psicología, que se ha centrado en la díada madre-hijo, resulta limitada (VALSINER, 2000); este modelo, que ha influenciado los enfoques de investigación del desarrollo, respondería a expectativas sociales europeas y norteamericanas sobre el rol (exclusivo) de la madre como cuidadora del bebé. En este sentido, el autor propone que los modelos que se han utilizado para entender las interacciones adulto-bebé derivan de una sobre generalización de una construcción cultural / colectiva de la clase media occidental, considerándola como una norma universal.

$\mathrm{Al}$ respecto, una revisión de la literatura científica de los últimos años permite apreciar que gran parte de la investigación sobre las interacciones de las y los bebés durante las últimas cuatro décadas ha tenido como foco de atención principal la interacción adulto-bebé, más específicamente madre-bebé, de la mano de enfoques como la teoría del apego y teorías psicodinámicas, encontrándose principalmente estudios relacionados con la lactancia, recién nacidos pretérmino, depresión materna y regulación emocional. Es el caso de Alvarenga, Malhado e Lins (2014), Bakeman y Brown (1980), Bystrova y otros autores (2009), Campbell, Cohn y Meyers (1995), Cohn y Tronick (1989), Egeland y Faber (1984), Kim y otros autores (2011), Shaw (1977), St James-Roberts, Conroy y Wilsher (1998) entre otros.

No obstante, durante los últimos años se ha ampliado la investigación a las relaciones entre pares, la que se encuentra dirigida en su mayoría a niños mayores de 18 meses (VASCONCELOS et al., 2003; ANJOS et al., 2004), lo que evidencia una carencia en la exploración de los fenómenos relacionales de los primeros meses de vida desde un enfoque interaccional complejo, desde una perspectiva situada, sensible a la diversidad de contextos culturales en donde se desarrollan los infantes (PIZARR0, 2013). Al respecto, cabe mencionar que los pocos estudios que indagan la relacionalidad en los primeros meses de vida desde esta perspectiva han sido desarrollados únicamente en Brasil, es el caso de Amorim, Vitoria, y Rossetti-Ferreira (2000), Amorim, Anjos y Rossetti-Ferreira (2012), Amorim y Rossetti-Ferreira (2008), Anjos y otros autores (2004) y Vasconcelos y otros autores (2003).

Se hace necesario, entonces, abordar los contextos que habita el sujeto en los primeros años de vida desde una perspectiva situada, atenta a las características locales. En la realidad latinoamericana, esto implica considerar el uso creciente de instancias de educación temprana; éstas se encuentran asociadas al aumento de la demanda de cuidados externos a la familia, así como a un mayor acceso a la educación temprana en la región (UNESCO, 2015; SEGUEL, 2012). Al respecto, cabe destacar la implementación progresiva de políticas de desfamiliarización de los cuidados en América Latina, entendidas como la delegación a servicios con participación estatal de las responsabilidades asociadas al cuidado, tradicionalmente asociadas a la familia, particularmente a la mujer (BLOFIELD; MARTÍNEZ, 2014). 
En Chile, país donde se desarrolla este estudio, este fenómeno se ha traducido en un aumento progresivo del gasto público y privado, logrando una mayor cobertura en educación preescolar, incluyendo a niñas y niños menores de 3 años (SEGUEL, 2012; ALARCÓN, 2015). A nivel institucional esta atención se encuentra estructurada en dos ciclos de educación parvularia que abarcan desde los primeros meses hasta el momento de ingresar a la educación básica (CHILE, 2005). El nivel inicial del primer ciclo es denominado sala cuna menor, donde son matriculados infantes hasta el año de vida.

En el contexto de la sala cuna, cada bebé se encuentra en un nuevo entorno social, conformado por la Educadora de Párvulos y los Técnicos en Educación Parvularia, así como por el grupo de pares que asiste regularmente al mismo espacio. Es bajo este contexto institucional que las niñas y los niños encuentran uno de sus principales escenarios de participación social antes de los 3 años de vida, donde los pares constituyen parte fundamental del proceso de socialización del niño o niña (TOMASINI, 2008).

A partir de lo expuesto, el presente trabajo se construye desde un enfoque sistémico y cultural del desarrollo humano (AMORIM, 2012; VALSINER, 2000), que supone el estudio de las interacciones que se despliegan en la primera etapa de la vida, más allá de la díada madre-hijo, contextualizado a los ambientes en donde el o la bebé se desenvuelve y las personas que constituyen parte de su entorno social inmediato, relevando el lugar de los pares como protagonistas del mundo de la y el bebé. Esto se aborda desde la comprensión de las relaciones entre pares en los primeros 18 meses de vida, como un fenómeno complejo, situado e histórico, que puede ser comprendido a través del análisis de las dinámicas relacionales en un contexto particular (ANJOS et al., 2004), el de la sala cuna.

Desde esta perspectiva, el objetivo del estudio que se presenta fue analizar el fenómeno de la relacionalidad y la construcción cultural que se despliega en la interacción con pares durante los primeros 18 meses de vida, en el contexto de la sala cuna.

Cabe mencionar, por último, que el presente documento hace parte de un trabajo de tesis (finalizado) de la primera autora, guiado por el segundo autor, desarrollado en una sala cuna ubicada en el sur de Chile.

\section{Metodología}

El estudio sobre el cual se construye el presente documento, se planteó como una investigación de tipo cualitativo, inspirada en la perspectiva propuesta por RossettiFerreira, Amorim, Soares-Silva y Oliveira (2008), denominada Redes de significado o RedSig. Ésta se construye utilizando la metáfora de red, para explicar las relaciones como un entramado heterogéneo e inseparable de interacciones, acciones, ocurrencias, que reconoce la contradicción e impredictibilidad en los fenómenos, abrazando la presencia de la incertidumbre y entendiendo la investigación como un proceso situado en contextos histórico-culturales. De esta forma, se fundamenta en el paradigma de la complejidad para aproximarse a la comprensión del desarrollo del bebé, mediante el análisis sistémico de elementos personales, relacionales y contextuales, que aborde un aspecto del fenómeno de estudio en profundidad, sin olvidar que existen otros niveles que participan de este entramado o RedSig. 
El estudio que se presenta, se planteó como estudio de caso, donde el grupo de sala cuna -esto es, bebés y equipo técnico pedagógico- fue considerado como el caso de estudio. Con esto se buscó explorar aspectos que no habían sido profundizados en otras investigaciones propuestas desde el enfoque de RedSig (AMORIM, 2012; ANJOS et al., 2004), cuyos resultados se centran en casos individuales, describiendo los patrones de interacción de sujetos específicos.

El estudio se desarrolló el año 2012, en una sala cuna menor ubicada en una ciudad del sur de Chile, y tomó los resguardos éticos correspondientes, que incluyeron el uso de consentimiento informado, tanto para la institución como para las y los participantes o sus representantes legales. Con el propósito de resguardar la confidencialidad, los nombres de cada participante fueron reemplazados por seudónimos.

Para efectos del estudio, se incorporaron las niñas y los niños cuyas familias consintieron en su participación y que presentaron asistencia regular durante el período de recolección de datos. De esta forma, el grupo consistió en cinco niñas y cuatro niños, además del equipo técnico pedagógico (una educadora de párvulos y dos técnicos en educación parvularia) y la madre de uno de los niños.

El proceso de investigación se desarrolló en tres fases sobrelapadas que permitieron la recolección y análisis de datos mediante un proceso recursivo y continuo.

La primera fase consistió en una recolección de datos inicial mediante sesiones de observación participante, en las que la primera autora se incorporó a las actividades cotidianas de la sala cuna, como una adulta más dentro de la sala. Estas tuvieron una duración de alrededor de noventa minutos cada una, y se realizaron durante la jornada de la mañana, durante tres meses. El primer mes tuvo una frecuencia de dos veces por semana, para luego descender a una sesión semanal; durante este periodo se suspendieron dos sesiones, por razones ajenas al estudio. Todas las sesiones fueron registradas de forma audiovisual, mediante equipo de grabación instalado en una de las esquinas de la sala, dado que para disminuir la intervención en el contexto, se decidió que la primera autora no recibiera asistencia externa en el manejo del equipo audiovisual.

A partir de los hallazgos de la observación participante, se definieron dos tipos de interacciones a analizar, los que fueron escogidos por su pertinencia respecto al enfoque y objetivos del estudio. Éstas fueron denominadas interacciones tipo $V$ y grupales. Las interacciones tipo $V$ se entendieron como aquellas en las que una o un bebé vértice interactúa con otras dos personas, pero éstas no interactúan entre sí. Por otra parte, se definieron como interacciones grupales aquellas en las que se involucran tres o más personas, incluyendo al menos una o un bebé; en éstas se identifican múltiples relaciones entre participantes. De esta forma, se descartaron para el análisis los episodios que sólo involucraron relaciones diádicas.

Sobre esta base, la información registrada audiovisualmente fue sistematizada utilizando el enfoque microgenético. Este enfoque se utiliza para para estudiar procesos de cambio en el desarrollo humano, mientras éstos ocurren, en lugar de investigar hitos o productos observables después de determinado cambio (LAVELLI et al., 2008). Con este propósito se busca una alta densidad de observaciones en un periodo relativamente corto (semanas, meses), preferentemente durante etapas del desarrollo donde ocurren rápidas transformaciones (LAVELLI et al., 2008). Esta estrategia se ha utilizado ampliamente para 
estudiar los procesos relacionales de primeros años vida desde una perspectiva histórica, en concordancia con el enfoque de RedSig (AMORIM; ROSSETTI-FERREIRA, 2008b; LAVELLI et al., 2008). En este caso, se realizó un mapeo de interacciones, aplicado a las primeras cinco sesiones, que permitió construir categorías emergentes. El mapeo implicó la transcripción de todos los episodios de interacción tipo $\mathrm{V}$ o grupales, las que se organizaron en una planilla Excel, de acuerdo a la fecha y código de la fracción de video. Para cada interacción se indicaron, además, los nombres de las personas involucradas y la duración del episodio.

La segunda fase consistió en la aplicación de entrevistas al equipo técnico pedagógico, sobre la base del ejercicio analítico desarrollado durante la primera fase. De esta forma, en primer lugar se propuso una entrevista grupal a la educadora de párvulos y las dos técnicos en educación parvularia, en la que se discutieron distintas escenas seleccionadas del registro audiovisual, recogiendo impresiones y discutiendo de manera informal los hallazgos preliminares de la y el investigador. Los aprendizajes obtenidos mediante este ejercicio guiaron, posteriormente, una entrevista en profundidad con la educadora.

Por último, la tercera fase se orientó a la construcción del análisis categorial definitivo, sobre la base de los hallazgos y de los ejercicios analíticos realizados en las primeras etapas. Esto supuso un proceso de triangulación de fuentes.

\section{Resultados}

Las relaciones dialógicas entre pares desplegadas en la sala cuna en estudio o sala cuna menor (SCM), varían a lo largo del año en forma, frecuencia e intensidad, de acuerdo al desarrollo individual de cada bebé, su experiencia en otros espacios y al proceso socio-histórico que involucra la convivencia en este contexto, en concordancia con lo propuesto por Amorim, Anjos y Rossetti-Ferreira (2012). Para propósitos del presente estudio, se describen tres categorias que dan cuenta de estas relaciones. Estas categorías han sido denominadas miradas, resonancia gestual y mediación.

En primer lugar, entendemos las miradas como un reconocimiento del otro/otra, identificable mediante el contacto y la atención visual sobre las acciones de pares y adultas, y sobre los objetos dispuestos en la SCM, en un contexto organizado y negociado culturalmente (VALSINER, 2000). Las miradas se identifican como un aspecto central a las dinámicas relacionales en el contexto de la SCM. Al respecto, la educadora reporta que las miradas, en conjunto con el llanto y los gestos faciales, son identificadas por el equipo técnico pedagógico (ETP) como las principales formas de expresión de los bebés pequeños, especialmente durante los primeros meses en la SCM.

Dada su relevancia en la interacción dentro de la SCM, las miradas pueden interpretarse como ejercicio fundamental para la construcción cultural en este contexto, constituyéndose en medio para el proceso de construcción de significados en esta etapa (AMORIM, 2012; CORREIA, 2009). Por una parte, la mirada a las interacciones entre pares y con adultas ${ }^{3}$, facilita la construcción de narrativas que van dibujando el mundo

3- En el contexto de estudio, todas las personas mayores presentes (equipo técnico pedagógico, madres e investigadora principal) son mujeres. 
cultural de las y los bebés, mediante un proceso activo de selección y significación de lo observado. Por otra, la mirada de cada bebé comunica a sus otros y otras, preferencias, miedos, éxitos, contribuyendo a la construcción colectiva de significados (CORREIA, 2009; VALSINER, 2000).

A medida que transcurre el año, si bien las miradas mantienen su rol protagónico en la interacción, van cambiando en forma y contenido. Durante los primeros meses, las miradas de las y los bebés se orientan principalmente hacia las adultas, de tal forma que se atiende visualmente a las y los pares sólo en momentos de cercanía física; estos momentos suelen ser iniciados por las y los bebés mayores, quienes se desplazan sin asistencia. En esta etapa, otro foco de atención visual son los materiales que las adultas entregan a cada bebé, quien además los explora mediante otros sentidos, destacando la alternancia entre la mirada y el tacto, al llevarlos a la boca. Este tipo de aproximación al material también se transforma con el tiempo, complejizándose en forma y duración.

En los siguientes meses, incluso antes de la aparición del desplazamiento independiente, las y los bebés comienzan a prestar mayor atención visual a sus pares, convirtiéndose en una acción cada vez más relevante dentro de las dinámicas relacionales de la SCM. Estas miradas se relacionan principalmente a situaciones de interacción entre bebés, bebé/adulto o acción sobre objeto, las que involucran secuencias de acción que incorporan una dialéctica entre la repetición y la novedad. Esto sugiere una construcción de significados mediante la narrativa de las acciones desplegadas en el contexto, coherente con lo propuesto por Bruner (2006). Esto se observa, por ejemplo, en la actividad del saludo, donde se realizan cantos, asociados a gestos y juegos con instrumentos musicales; lo mismo ocurre cuando un grupo de bebés se desplaza por la sala o disputan un juguete.

Por otra parte, las y los bebés continúan durante todo el año orientando su mirada hacia las adultas frente a situaciones particulares; frente al estrés, como el ingreso a la sala de un adulto desconocido o al recibir un golpe de un par; en las instancias donde se desafían los límites establecidos por las adultas; y frente a los logros, como completar una tarea autoimpuesta. Esta distinción que las y los bebés hacen en el foco de su mirada, sugiere la construcción de significados diversos en relación a pares y adultas. Al mismo tiempo, los procesos de transformación que se identifican en las miradas, constituyen un claro ejemplo de la dimensión histórica de la relaciones dialógicas dentro de la SCM (AMORIM; ROSSETTI-FERREIRA, 2008a; BAJTÍN, 1999).

Como segundo punto, imbricada con la mirada se encuentra la categoría denominada resonancia gestual. Esta hace referencia a la coordinación de acciones, ya sea inmediata o mediata, entre pares o con adultas, que se entiende a partir de la noción de campo interactivo descrita anteriormente (ANJOS et al., 2004). Cabe mencionar que, similar al caso de las miradas, la resonancia gestual no se produce indiscriminadamente; en relación a las adultas, la resonancia gestual de niñas y niños aparece en relación a actividades asociadas a las rutinas de cuidado, como mecer una silla o entregar un biberón. Por otra parte, en relación a los pares, la resonancia gestual se asocia a la exploración y al juego, como se grafica en un extracto ${ }^{4}$ del mapeo.

4- Para facilitar la lectura de las citas al material de estudio, se utilizan las siglas RA (registro audiovisual de acciones en el aula), CE (comunicación personal, entrevista en profundidad con educadora, en otras dependencias de la sala cuna) y CETP (Comunicación personal, 
Esteban comienza a manipular el material con que trabajan Isabel y Teresa (adulta). Magda asume la postura del oso, Isabel y Esteban interrumpen su acción y miran detenidamente a Magda; las adultas rien. Esteban gatea hacia Magda y mira su rostro. Isabel también asume la postura del oso. Otra adulta comenta que Isabel ha hecho lo mismo que Magda. (RA, 27 agosto).

En este caso, Isabel hace eco de la postura de Magda, sin que ella la mire o dé señales de percatarse de esta acción. No obstante, ambas niñas y Esteban, quien también participa de esta escena, han estado interactuando minutos antes, dando cuenta de su involucramiento en un campo interactivo.

La resonancia gestual, en este sentido, constituye un eje relacional y de construcción de significados entre pares de la SCM, al mismo tiempo que se constituye en mediador en la construcción del sujeto y su corporiedad (AMORIM; ROSSETTI-FERREIRA, 2008b; VALSINER, 2000). Al respecto, la educadora sugiere que las o los bebés mayores representan modelos a seguir para las y los más pequeños, quienes aprenden mediante la observación. Como ejemplo, propone la adquisición de la marcha; plantea que, una vez que la primera niña comenzó a caminar, las y los otros la observaron y luego intentaron repetir la acción, hasta seguirla. "El hecho de que observen que los otros lo están haciendo, los impulsa a tratar de hacerlo, también” (CE, 26 diciembre).

La dinámica relacional entre pares es incentivada y enriquecida por la acción adulta. En este marco se van generando nuevas instancias de diálogo, que no se limitan a la relación entre dos personas, sino que involucra a otras y otros participantes del entorno social, tanto bebés como adultas (AMORIM; ROSSETTI-FERREIRA, 2008a). En este sentido, el rol de iniciar la acción que conduce a la resonancia gestual varía de una instancia a otra, pudiendo ser una adulta, una o un bebé, o el resultado de una acción conjunta. Estas interacciones, como se discutió anteriormente, evidencian la construcción del sujeto mediada por la cultura. A este respecto, la mediación directa del ETP es frecuente.

Paula (adulta) está jugando con Francisca, usando el teléfono; Alonso está sentado cerca de la niña y altera miradas entre Paula y Francisca, entre acción y juguete. Paula pone el auricular en la oreja de la niña. Ella realiza el gesto de sostener el auricular junto a su oreja. Otra adulta dice "aló, Fran", Francisca le entrega el auricular. Alonso vocaliza y extiende el auricular hacia la otra adulta; luego lo lleva a su boca. Francisca recibe el auricular de regreso; Paula dice aló y ella repite el gesto; Alonso vocaliza. Francisca entrega el auricular nuevamente. Alonso lleva el auricular a su oreja y balbucea "ala”. Cada niño continúa explorando su juguete. (RA, 9 agosto).

Isabel, Magda y Esteban tienen cada uno un material de encaje. Las niñas miran frecuentemente el material de su compañera y del niño. Teresa (adulta) ayuda a Magda ubicar las piezas y la niña participa, Isabel mira la escena. Esteban muestra una pieza a su mamá. Luego Teresa ayuda a Isabel y la niña participa, mientras Magda mira la escena y luego toca el material de Isabel, quien le muestra una pieza a Teresa; luego Magda levanta una pieza. Magda toca el material de Isabel [...] ambas niñas tocan el material de Magda. (RA, 27 agosto).

entrevista con equipo técnico pedagógico, en otras dependencias de la sala cuna). A cada sigla le sigue el día y el mes en que se realizó el registro (todos durante el año 2012). 
La mediación constituye la tercera categoría de análisis del presente estudio. Ésta es entendida como la participación adulta en relación a las interacciones entre pares en el contexto de la SCM. En este sentido, las acciones adultas son comprendidas en su rol de mediador semiótico (VALSINER, 2000), que dan cuenta de diversos aspectos sociales e históricos, tales como la estructura y política institucionales, estilos personales y educación formal del ETP; al mismo tiempo, implican la construcción y organización mediada culturalmente de los espacios y los tiempos, que incluye la infraestructura de la sala cuna y el jardín infantil, así como la distribución del mobiliario y los objetos, organización de la jornada, entre otros. Estos aspectos se relacionan en un entramado de significados culturales, explícitos e implícitos, que se evidencian en lo cotidiano, encontrado en los sentidos comunicados por el ETP y en su correlato en las acciones observables durante la jornada.

En este sentido, cabe mencionar que el rol que el ETP atribuye a la sala cuna como instancia educativa, se identifica como un elemento guía para las acciones de mediación desplegadas por las adultas. Al respecto, el equipo describe la sala cuna como oportunidad para participar en un contexto enriquecido desde una perspectiva social y material, con el propósito de alcanzar el desarrollo integral de las y los bebés. Con este objetivo se organiza el espacio y se diseñan actividades específicas se encuentran la adquisición de hitos motores y verbales, así como el desarrollo de habilidades para la convivencia, donde las conductas entre pares son foco de la atención adulta.

Entre las interacciones que convocan mayor atención del ETP, se encuentran las situaciones de conflicto entre pares, dada la frecuencia y relevancia que éstas implican. Estas demandan una serie de acciones por parte del equipo, orientadas a evitar o prevenir el conflicto y las conductas asociadas, como golpes o empujones. En este sentido, la acción adulta da cuenta de los significados construidos en relación al conflicto, los que tienen a evaluarse negativamente.

Si se pegan con intención, nosotros tenemos que llevarlo, [...] 'hagámosle cariño para que no llore', eso del 'nanai' (acariciar) si funciona; pero uno los tiene que llevar, todavía no reaccionan al llanto del otro, están más interesados en haber ganado. Pero si uno los lleva a la situación, ahí le hacen cariño, un poco como induciéndolos a eso, no muy voluntario (ríe). (CE, 26 diciembre).

A este respecto, frecuentemente se encuentran situaciones de conflicto que mantienen una estructura común, constituida por tres elementos básicos; primero, la presentación del conflicto, entendida como el momento en que una bebé inicia una acción potencialmente desagradable o transgresora hacia otra; segundo, la mediación directa, momento en el que una o más adultas indica a la bebé que dicha acción no es aceptada y propone una nueva, hacer nanai (acariciar); tercero, desenlace, que puede consistir en la insistencia de la bebé en la primera acción o en la aceptación de la propuesta adulta. En el primer caso, la adulta modifica su abordaje mediante estrategias como desviar la atención de la bebé que inicia la secuencia, limitar el movimiento de una o ambas bebés o distanciar a las pares involucradas. En el segundo caso, si bien el o la bebé acaricia a su par, muchas veces inicia la secuencia poco tiempo más tarde. 
Dada la complejidad inherente a las relaciones dialógicas, cabe destacar que estas secuencias no se repiten en patrones rígidos, sino que aparecen como combinaciones de los elementos descritos, repeticiones de acciones y variaciones individuales. Esta diversidad dice relación con la edad y estados emocionales de las y los bebés involucrados, las características personales y el aspecto histórico de la dinámica relacional de las y los pares en conflicto. Otra variación encontrada dice relación con el estilo de mediación de cada adulta, principalmente en la forma de anticiparse a una potencial interacción conflictiva. Así, ante la aproximación de dos bebés con historia de conflicto algunas adultas advierten nanai y observan la situación antes de intervenir, mientras que otras tienden a evitar el contacto entre estos pares.

Ceci trae pilares de argollas e invita a los niños y niñas a jugar. Alonso comienza a jugar con una, Francisca se acerca, la toma y la aleja de Alonso, él se queja y mira a Teresa quien se está sentando junto a él. La adulta dice "compartir, compartir" y Alonso, aún con una argolla en la mano, vuelve a mirarla y a quejarse. Isabel llega gateando. Ceci trae nuevos pilares y devuelve a Alonso el suyo, con el que comienza a jugar. Sienta a los niños en círculo y distribuye el material. Cada niño juega con un pilar de argollas. Julieta deja su pilar a un lado y le quita su material a Isabel, quien lo recupera. Julieta se mece y agita los brazos, y Ceci le entrega nuevamente el material que le corresponde. Luego Isabel toma el pilar de Julieta y se lo entrega a Ceci, quien lo devuelve a su dueña. (RA, 16 agosto).

Magda intenta tomar el juguete de Isabel, quien le da manotazos en el brazo; Teresa (adulta) dice "nanai, nanai, besito" y baja la mano de Isabel; Magda se inclina hacia Isabel, estirando los labios; ella no la ve. Magda repite intento con el juguete y ambas forcejean suavemente; Teresa repite "nanai" [...] Magda se acerca al juguete de Isabel y Teresa la aleja. (RA, 21 agosto).

Con el paso del tiempo, la interacción entre pares se hace más variada, observándose diversas narrativas frente a situaciones potenciales de conflicto. Al respecto, se encuentra que las niñas y los niños incorporan acciones vivenciadas a través de su historia relacional, como la expresión de caricias y besos entre pares sin la intervención de las adultas. Al mismo tiempo, las adultas transforman sus prácticas sobre la base de la historia compartida con niñas y niños. Esto da cuenta de un proceso de transformación de la relación entre pares, desarrollada a partir de la historia dialógica en el contexto de la SCM, donde unas voces hacen eco de otras (BAJTÍN, 1999). Al mismo tiempo, este proceso refleja la negociación de significados entre adultas y bebés, que suele orientarse a la aceptación social del nanai por sobre el acto de golpear.

Isabel se agacha y besa a Loreto en la cara y luego se levanta; Ceci (adulta) sonríe. Isabel repite esta acción varias veces, también la acaricia en algunas ocasiones. Ceci dice, "huy, que rico beso". Isabel se inclina nuevamente sobre Loreto y la besa. Ceci, sonriendo, comenta que Loreto estira los labios cuando la besan. Isabel se acerca a Ceci, ella la acaricia y a Loreto. Loreto, mira detenidamente a Ceci; mantiene su mano en una pierna de la adulta e Isabel en la otra. (RA, 27 agosto). 
Por otra parte, la habilidad de las bebés para trasladarse en la sala, permite la aparición de secuencias complejas de interacción en relación al material, dando mayor protagonismo al espacio como mediador de las situaciones de interacción entre pares. Éste cobra especial relevancia en las dinámicas relacionales entre bebés grandes, asociándose a situaciones de resonancia gestual donde niñas y niños se desplazan hacia los mismos rincones que sus compañeros, de manera que se generan puntos preferidos dentro de la sala, donde las y los bebés suelen desplazarse (CETP, 28 septiembre).

\begin{abstract}
Magda juega en rincón de la tecnología, se acerca Esteban caminando de la mano de su mamá; Esteban toma un juguete y Magda mira Esteban-juguete-adulta-Esteban. Él se aleja caminado de la mano; Magda lo mira. Esteban la mira y luego vuelve al rincón, le toca la espalda y tira el brazo de su compañera, ambos se miran detenidamente. (RA, 27 agosto).
\end{abstract}

Este tipo de interacciones es estimulada y/o tensionada por las acciones del ETP, quienes orientan las relaciones desplegadas en el espacio, a través de la organización de la sala y de acuerdo a los distintos momentos planificados para la jornada. En este sentido es frecuente la agrupación de niñas y niños en círculo o distribuciones similares, sentados sobre una colchoneta. Esta distribución favorece el contacto visual entre pares y la interacción mediada por objetos, mientras disminuye el desplazamiento por la sala. En este sentido, cabe destacar que los objetos se constituyen en puntos de encuentro y en elemento de conflicto.

\begin{abstract}
Loreto arroja un cascabel en la caja (...), Magda se agacha, golpea la caja y luego la toma. Loreto intenta echar el juguete, pero Madga está moviendo la caja, y no lo logra; entonces Loreto se queja. Luego Magda la pone en el suelo y Loreto gatea hacia la caja, alcanzándola y acercándola a sí. Magda se sienta y ambas niñas juegan con la caja. Comienzan a jalarla ambas, Loreto se queja y la tira con fuerza; saca un juguete y Magda se queda con la caja. (RA, 21 agosto).
\end{abstract}

\title{
Discusión
}

El presente estudio se propuso analizar el fenómeno de la relacionalidad y la construcción cultural que se despliega en la interacción con pares durante los primeros 18 meses de vida, en el contexto de la sala cuna. A partir de este análisis, se exponen a continuación las principales conclusiones.

En primer lugar, en coherencia con lo expuesto por Amorim y Rossetti-Ferreira (2008a; 2008b) el análisis de la relacionalidad desplegada en el contexto de la sala cuna, permite aproximarse a la riqueza y complejidad de los procesos de construcción cultural, desde edades muy tempranas. Se destaca, en este sentido, el lugar que ocupan las interacciones entre pares en la construcción de significados de cada bebé, y su distinción con lo ocurrido en el marco de la relacionalidad adulta/bebé. En este contexto, los pares ofician como agentes culturales, que participan de la mediación entre sus compañeras y compañeros y las experiencias en el espacio, con los objetos y con los otros. De esta forma la resonancia gestual y la mirada constituyen no sólo un eje relacional, sino 
de construcción de cultura e incorporación de significados compartidos entre pares, favoreciendo el despliegue de nuevos aprendizajes.

Bajo esta perspectiva, el estudio releva la dimensión socio-histórica de la relación dialógica (BAJTÍN, 1999), dado que los procesos de construcción compartida de significados solo son apreciables en el tiempo, a través de la narrativa constituida por las diversas interacciones, breves en un comienzo, más complejas con el curso de los meses (AMORIM, 2012; BRUNER, 2006).

Otro aspecto significativo de la relacionalidad en la sala cuna estudiada, consiste en el rol adulto respecto a las interacciones entre pares. En este sentido, se identifica la relevancia de la acción adulta en la mediación semiótica, como acto dialógico que va transmitiendo-actualizado-construyendo cultura en el devenir de las diversas situaciones de interacción. De esta forma, se encuentran situaciones de acción adulta intencionada hacia contenidos específicos, como el uso del no, nanai frente al conflicto. Esta acción permite construcción de significados (CORREIA, 2009) evidenciados en la evolución histórica de las interacciones entre pares, apreciadas en la aparición de caricias y besos sin la necesidad de indicación directa, lo que constituye un ejemplo de construcción de significados culturales en el proceso relacional. Esto es coherente con los hallazgos de Tomasini (2008), quien en su estudio en sobre procesos de socialización de niños y niñas mayores de 3 años en contexto preescolar, encontró que las características de la mediación de los adultos frente al conflicto - o la falta de ésta - perfilan la experiencia y aprendizajes en este contexto, la que puede variar desde oportunidades para desarrollar estrategias de manejo de conflicto, hasta la vivencia repetitiva de situaciones de violencia y disminución de los recursos para enfrentar la vida escolar.

Por otra parte, se encuentran situaciones que pueden definirse como mediación no intencionada, entendidas como aquellas en que la adulta interactúa con un o una bebé y es mirada por otro $u$ otra, participando de un campo interactivo (ANJOS et al., 2004) que media la relación dialógica entre pares. Esta variedad en las dinámicas relacionales abre un espacio a la diversidad de experiencias en el aula, ampliando el aprendizaje y enriqueciendo la construcción de cultura.

Los resultados de la presente investigación, entonces, permiten entender los procesos de desarrollo y aprendizaje como fenómenos complejos, dialógicos, que implican la interacción con otros sociales de diversas edades, mediante los procesos de resonancia gestual, mirada y mediación.

Esto pone sobre la mesa nuevos elementos que enriquecen la discusión acerca de la pertinencia del primer ciclo de educación parvularia, particularmente en relación a su contraste con las corrientes que abogan por los cuidados maternos exclusivos durante los primeros tres años de vida, fundamentadas principalmente en la teoría de apego. Pues, si bien la vasta investigación que sostiene la relevancia de la díada madre/hijo no pretende ser negada en el presente documento, los hallazgos de la investigación que se presenta apuntan a la relevancia de la interacción con pares, desde las primeras etapas de la vida, quienes no necesariamente se encuentran en el contexto de un hogar nuclear. Se levanta así, la idea de la sala cuna como un posible espacio de encuentro con pares; otro contexto para la construcción de cultura. 
Consideramos, por otra parte, que el estudio del desarrollo temprano demanda profundización de los aspectos socio-históricos, culturales y espaciales donde éste se despliega, en concordancia con lo propuesto por Valsiner (2000). En este sentido, cabe destacar la riqueza del análisis microgenético como método análisis de datos y la pertinencia de profundizar este análisis mediante estrategias etnográficas. La sinergia entre ambas permite visibilizar la dimensión socio-histórica de las dinámicas relacionales entre el contexto de estudio, aproximándose a la comprensión de la construcción de significados en tanto narrativa (BRUNER, 2006). Esto resulta particularmente necesario en la aproximación al fenómeno de la construcción de significados, en sujetos que aún no se expresan mediante lenguaje verbal (AMORIM, 2012).

Como afirmación final, se considera relevante destacar que la presente investigación, en su calidad de estudio de caso, refleja la experiencia situada de un grupo de personas, en un contexto espacio temporal determinado, por lo tanto, no busca establecer una teoría general sobre el fenómeno estudiado. No obstante, para los autores del presente documento, la experiencia expuesta puede encontrar resonancia en otras aulas o espacios de interacción en la etapa preescolar; de tal forma, el estudio que se presenta se propone como una apertura al diálogo sobre el fenómeno de la relacionalidad entre pares en los primeros años de vida.

\section{Referencias}

ALARCÓN, Jorge et al. Desafíos de la educación preescolar en Chile: ampliar la cobertura, mejorar la calidad y evitar el acoplamiento. Estudios Pedagógicos, Valdivia, v. 41, n. 2, p. 287-303, 2015.

ALVARENGA, Patricia; MALHADO, Sâmia de Carliris; LINS, Taiane Costa de Souza. 0 impacto da responsividade materna aos oito meses da criança sobre as práticas de socialização maternas aos 18 meses. Estudos de Psicologia, Natal, v. 19, n. 4, p. 305-314. out./dez. 2014.

AMORIM, Katia de Souza. Processos de significaçãoo no primero ano de vida. Psicologia: teoria e pesquisa, Ribeirão Preto, v. 28, n. 1, p. 45-53, jan./mar. 2012.

AMORIM, Katia de Souza; ANJOS, Adriana Mara dos; ROSSETTI-FERREIRA, Maria Clotilde. Processos interativos de bebês em creche. Psicologia: teoria e pesquisa, Porto Alegre, v. 25, n. 2, p. 378-389, 2012.

AMORIM, Katia de Souza; ROSSETTI-FERREIRA, Maria Clotilde. Corporeidade, significação e o primeiro ano de vida. Arquivos Brasileiros de Psicologia, Rio de Janeiro, v. 60, n. 1, p. 67-81, 2008b.

AMORIM, Katia de Souza; ROSSETTI-FERREIRA, Maria Clotilde. Dialogismo e a investigação de processos desenvolvimentais humanos. Paidéia, Ribeirão Preto, v. 18, n. 40, p. 235-250, 2008a.

AMORIM, Katia de Souza; VITORIA, Telma; ROSSETTI-FERREIRA, Maria Clotilde. Rede de significações: perspectiva para análise da inserção de bebês na creche. Cadernos de Pesquisa, São Paulo, v. 109, n. 3, p. 115-144, 2000.

ANJOS, Adriana Mara et al. Interações de bebês em creche. Estudos de Psicologia, Natal, v. 9, n. 3, p. 513-522, 2004. 
BAJTín, Mijail. Estética de la creación verbal. 10. ed. Madrid: Siglo Veintiuno, 1999.

BAKEMAN, Roger; BROWN, Josephine. Early interaction: consequences for social and mental development at three years. Child Development, Ann Arbor, v. 51, n. 2, p. 437-447, 1980.

BLOFIELD, Merike; MARTíNEZ, Juliana. Trabajo, familia y cambios en la política pública en América Latina: equidad, maternalismo y corresponsabilidad. Revista Cepal, México, D.F., n. 114. p. 107-125, dic. 2014.

BRUNER, Jerome. Actos de significado: más allá de la revolución cognitiva. Madrid: Alianza, 2006.

BYSTROVA, Ksenia et al. Early contact versus separation: effects on mother-infant interaction one year later. Birth, Hoboken, v. 36, n. 2, p. 97-109, 2009.

CAMPBELL, Susan; COHN, Jeffrey; MEYERS, Teri. Depression in first-time nothers: mother-infant interaction and depression chronicity. Developmental Psychology, Washington, v. 31, n. 3, p. 349-357, 1995.

CHILE. Ministerio de Educación. Bases curriculares de educación parvularia. Santiago de Chile: ME, 2005.

COHN, Jeffrey; TRONICK, Edward. Specificity of infants' response to mothers' affective behaviour. Journal of the American Academy of Child and Adolescent Psychiatry, New York, v. 28, n. 2, p. 242-248, 1989.

CORREIA, Mônica. Indeterminação, multidimensionalidade e relevância do processo de Construção de significados. Psicologia em Estudo, Maringá, v. 14, n. 2, p. 251-258, abr./jun. 2009.

EGELAND, Byron; FARBER, Ellen. Infant-mother attachment: factors related to its development and changes over time. Child Development, Ann Arbor, v. 55, n. 3, p. 753-771, 1984.

KIM, Pilyoung et al. Breastfeeding, brain activation to own infant cry, and maternal sensitivity. Journal of Child Psychology and Psychiatry and Allied Disciplines, v. 52, n. 8, p. 907-915, 2011.

LAVELLI, Manuela et al. Using microgenetics desings to study change processes. In: TETI, Douglas. Handbook of research methods in developmental science. Oxford: Blackwell, 2008. p. 40-65.

PIZARRO, Eugenia. Relaciones dialógicas y construcción de significados en el contexto de la sala cuna. 2013. 120 p. Tesina (Magíster en Comunicación) - Facultad de Filosofía y Humanidades, Universidad Austral de Chile, Valdivia, 2013.

ROSSETTI-FERREIRA, Maria Clotilde et al. Desafios metodológicos na perspectiva da rede de significações. Cadernos de Pesquisa, São Paulo, v. 38, n. 133, 147-170, 2008.

SCORSOLINI-COMIN; Fabio; SANTOS, Manoel Antônio. Bakhtin e os processos de desenvolvimento humano. Revista Brasileira de Crescimento e Desenvolvimento Humano, São Paulo, v. 20, n. 3, p. 745-756, 2010.

SEGUEL, Ximena et al. ¿Qué efecto tiene asistir a sala cuna y jardín infantil desde los tres meses hasta los cuatro años de edad?: Estudio longitudinal en la junta nacional de jardines infantiles. Psykhe, Santiago, v. 21, n. 2, p .87-104, nov. 2012. 
SHAW, Celia. A comparison of the patterns of mother baby interaction for a group of crying, irritable babies and a groups of more amenable babies. Child, Hoboken, v. 3, n.1, p. 1-12, 1977.

ST JAMES-ROBERTS, lan; CONROY, Susan; WILSHER, Katie. Links between maternal care and persistent infant crying in the early months. Child, Hoboken, v. 24, n. 5, p. 353-376, 1998.

TOMASINI, Marina. La escolaridad incial como contexto socializador: complejidad y conflictividad en la trama interactiva cotidiana. Revista Mexicana de Investigación Educativa, México, v. 13, n. 36, p. 7-34, ene./mar. 2008.

UNESCO. La educación para todos, 2000-2015: logros y desafíos. París: Organización de las Naciones Unidas para la Educación, la Ciencia y la Cultura, 2015.

VALSINER, Jaan. Comparative study of human cultural development. Madrid: Fundación Infancia y aprendizaje, 2001.

VALSINER, Jaan. Culture and human development. London: Sage, 2000.

VASCONCELOS, Cleido Roberto Franchi e et al. A Incompletude como virtude: interação de bebês na creche. Psicologia: Reflexão e Crítica, Porto Alegre, v. 16, p. 2, p. 293-301, 2003.

Recibido en :09.03.2016

Aprobado en: 09.08.2016

Eugenia Paz Pizarro Troncoso es académica en la Facultad de Medicina de la Universidad Austral de Chile. Terapeuta ocupacional (Universidad de Chile). Magíster en comunicación, Universidad Austral de Chile. Licenciada en ciencias de la Ocupación Humana, Universidad de Chile.

Sergio Toro Arévalo es profesor de educación física en la Pontificia Universidad Católica de Valparaíso. Doctorado en Ciencias de la Educación (Pontificia Universidad Católica de Chile). Académico en la Facultad de Filosofía u Humanidades de la Universidad Austral de Chile. 